\title{
Intrathyroidal Parathyroid
}

National Cancer Institute

\section{Source}

National Cancer Institute. Intrathyroidal Parathyroid. NCI Thesaurus. Code C96188.

A parathyroid gland embedded within the thyroid gland parenchyma. 